Supporting Information:

\title{
Effect of Non-Uniform Mass Transport on Nanobubble Nucleation at Individual Pt Nanoparticles
}

\author{
Nicholas S. Georgescu, Donald A. Robinson, and Henry S. White* \\ Department of Chemistry, University of Utah \\ *Corresponding author: white@,chem.utah.edu
}

\section{Comsol Model Report 2 of 3:}

\section{Steady State Limiting Current for Nanoparticle in SECCM Cell}

Below is an automatically generated Comsol model report, which describes in detail the parameters, boundary conditions, geometry, and study settings used in the finite element simulations. This model is used to estimate the limiting current for the nanoparticle within the pipet and establish the relationship between $i_{n b}^{p}$ and $c_{c r i t}$.

\section{Contents}

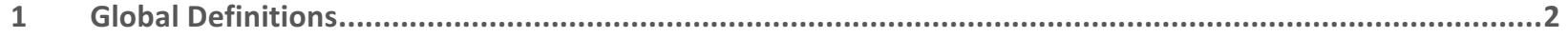

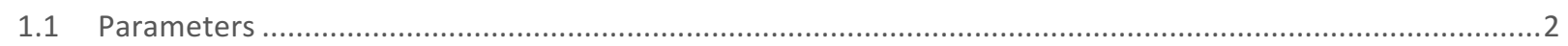

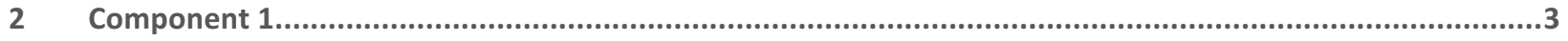

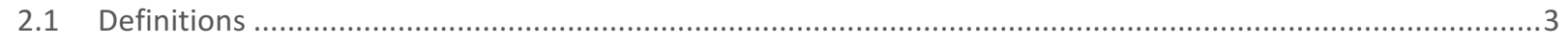

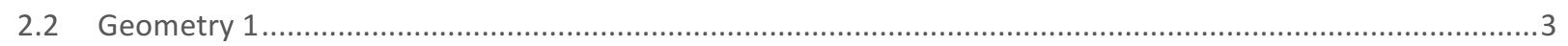

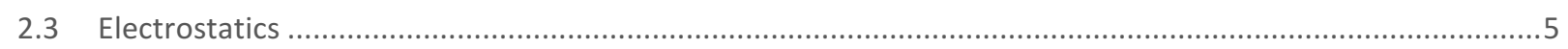

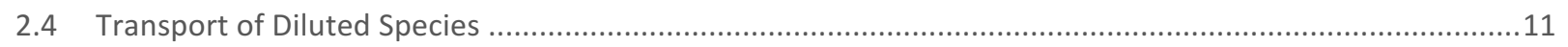

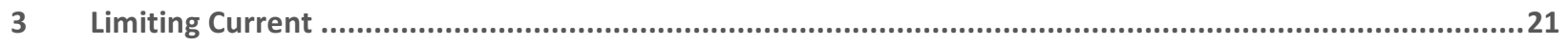

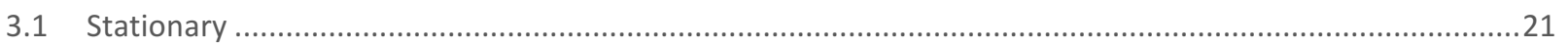

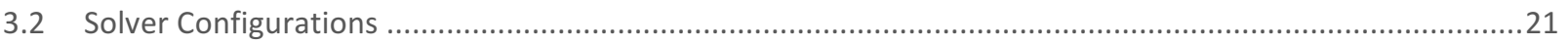




\section{Global Definitions}

USED PRODUCTS

COMSOL Multiphysics

Chemical Reaction Engineering Module

\subsection{PARAMETERS}

PARAMETERS 1

\begin{tabular}{|l|l|l|l|}
\hline Name & Expression & Value & Description \\
\hline $\mathrm{DH}$ & $7 \mathrm{E}-5\left[\mathrm{~cm}^{\wedge} 2 / \mathrm{s}\right]$ & $7 \mathrm{E}-9 \mathrm{~m}^{2} / \mathrm{s}$ & diffusion coefficient of protons \\
\hline $\mathrm{DH} 2$ & $4.5 \mathrm{E}-5\left[\mathrm{~cm}^{\wedge} 2 / \mathrm{s}\right]$ & $4.5 \mathrm{E}-9 \mathrm{~m}^{2} / \mathrm{s}$ & diffusion coefficient of $\mathrm{H} 2$ \\
\hline $\mathrm{DClO} 4$ & $1 \mathrm{e}-5\left[\mathrm{~cm}^{\wedge} 2 / \mathrm{s}\right]$ & $1 \mathrm{E}-9 \mathrm{~m}^{2} / \mathrm{s}$ & diffusion coefficient of perchlorate \\
\hline rnp & $35[\mathrm{~nm}]$ & $3.5 \mathrm{E}-8 \mathrm{~m}$ & radius of the nanoparticle \\
\hline cbulk & $500[\mathrm{mM}]$ & $500 \mathrm{~mol} / \mathrm{m}^{3}$ & bulk concentration \\
\hline theta & 7 & 7 & angle of the pipet in degrees \\
\hline hpipet & $5[\mathrm{um}]$ & $5 \mathrm{E}-6 \mathrm{~m}$ & height of the glass of the pipet \\
\hline rpipet & $600[\mathrm{~nm}]$ & $6 \mathrm{E}-7 \mathrm{~m}$ & radius of the pipet \\
\hline hdroplet & rpipet $/ 2$ & $3 \mathrm{E}-7 \mathrm{~m}$ & height of the droplet \\
\hline rbubble & rpipet/4 & $1.5 \mathrm{E}-7 \mathrm{~m}$ & radius of the bubble formed on the nanoparticle \\
\hline fillet & $1 / 200$ & 0.005 & how much of the model is cut into \\
\hline
\end{tabular}




\section{Component 1}

SETTINGS

\begin{tabular}{|l|l|}
\hline Description & Value \\
\hline Unit system & Same as global system \\
\hline Avoid inverted elements by curving interior domain elements & Off \\
\hline
\end{tabular}

\subsection{DEFINITIONS}

\subsubsection{Coordinate Systems}

Boundary System 1

\begin{tabular}{|l|l|}
\hline Coordinate system type & Boundary system \\
\hline Tag & sys1 \\
\hline
\end{tabular}

COORDINATE NAMES

First Second Third

\begin{tabular}{ll|l}
$\mathrm{t} 1$ & to & $\mathrm{n}$
\end{tabular}

\subsection{GEOMETRY 1}

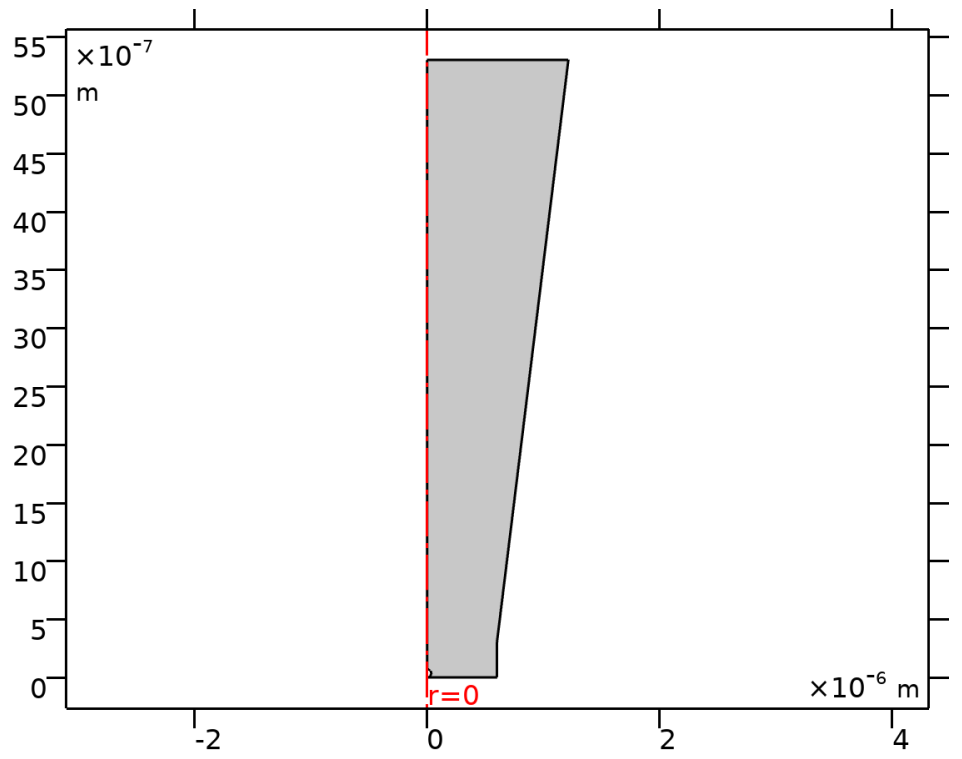

Geometry 1

UNITS

Length unit $\mathrm{m}$

Angular unit deg

GEOMETRY STATISTICS

Description Value 


\begin{tabular}{|l|l|}
\hline Description & Value \\
\hline Space dimension & 2 \\
\hline Number of domains & 1 \\
\hline Number of boundaries & 7 \\
\hline Number of vertices & 7 \\
\hline
\end{tabular}

\subsubsection{Polygon 1 (pol1)}

\section{OBJECT TYPE}

\begin{tabular}{|l|l|}
\hline Description & Value \\
\hline Type & Solid \\
\hline
\end{tabular}

COORDINATES

\begin{tabular}{|l|l|}
\hline Description & Value \\
\hline Data source & Table \\
\hline
\end{tabular}

COORDINATES

\begin{tabular}{|l|l|}
\hline $\mathbf{r}(\mathbf{m})$ & $\mathbf{z}(\mathbf{m})$ \\
\hline 0 & rnp*fillet \\
\hline 0 & hpipet+hdroplet \\
\hline rpipet+tan(theta*pi/180)*hpipet & hpipet+hdroplet \\
\hline rpipet & hdroplet \\
\hline rpipet & 0 \\
\hline rnp*fillet & 0 \\
\hline rnp*fillet & rnp*fillet \\
\hline 0 & rnp*fillet \\
\hline
\end{tabular}

\subsubsection{Circle 1 (c1)}

POSITION
\begin{tabular}{|l|l|}
\hline Description & Value \\
\hline Position & $\{0$, rnp $\}$ \\
\hline
\end{tabular}

ROTATION ANGLE

\begin{tabular}{|l|l|}
\hline Description & Value \\
\hline Rotation & -90 \\
\hline
\end{tabular}

SIZE AND SHAPE

\begin{tabular}{|l|l|}
\hline Description & Value \\
\hline Radius & $\mathrm{rnp}$ \\
\hline Sector angle & 180 \\
\hline
\end{tabular}




\subsubsection{Difference 1 (dif1)}

COMPOSE

\begin{tabular}{|l|l|}
\hline Description & Value \\
\hline Repair tolerance & Automatic \\
\hline
\end{tabular}

\subsubsection{Form Union (fin)}

SETTINGS

\begin{tabular}{|l|l|}
\hline Description & Value \\
\hline Repair tolerance & Automatic \\
\hline
\end{tabular}

\subsection{ELECTROSTATICS}

USED PRODUCTS

COMSOL Multiphysics

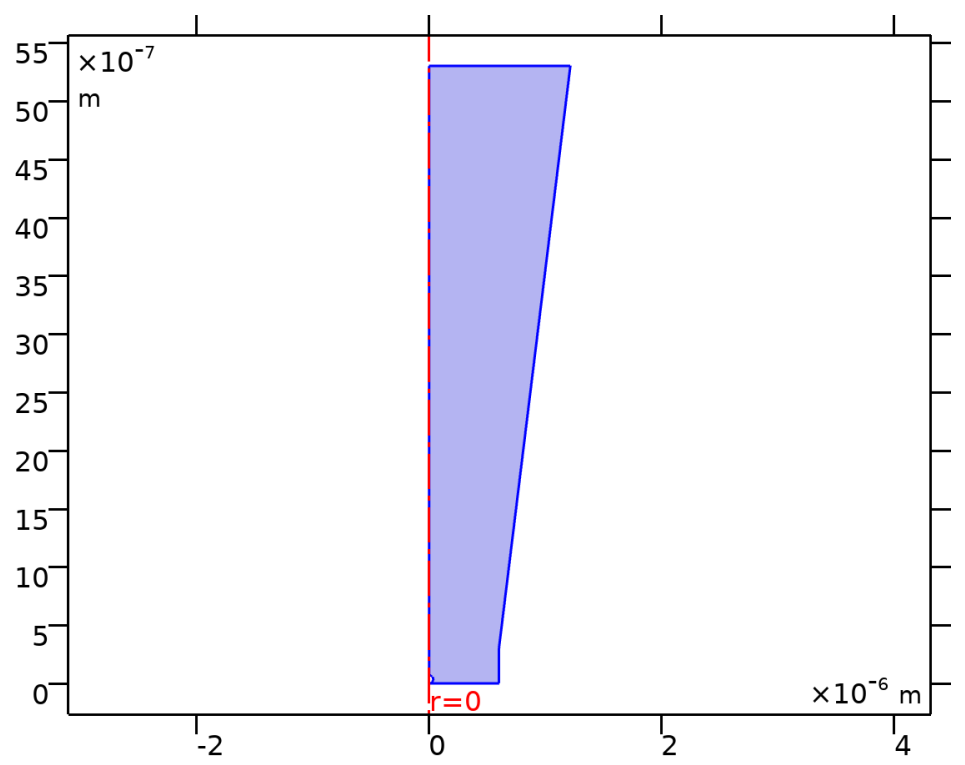

\section{Electrostatics}

\section{SELECTION}

Geometric entity level Domain

Selection Geometry geom1: Dimension 2: All domains

EQUATIONS

$$
\begin{aligned}
& \nabla \cdot \mathbf{D}=\rho_{V} \\
& \mathbf{E}=-\nabla V
\end{aligned}
$$




\subsubsection{Interface settings}

Discretization

SETTINGS

\begin{tabular}{|l|l|}
\hline Description & Value \\
\hline Electric potential & Quadratic \\
\hline
\end{tabular}

Manual terminal sweep settings

SETTINGS

\begin{tabular}{l|l}
\hline Description & Value \\
\hline Use manual terminal sweep & Off \\
\hline Reference impedance & $50[\mathrm{ohm}]$ \\
\hline
\end{tabular}

\subsubsection{Charge Conservation 1}

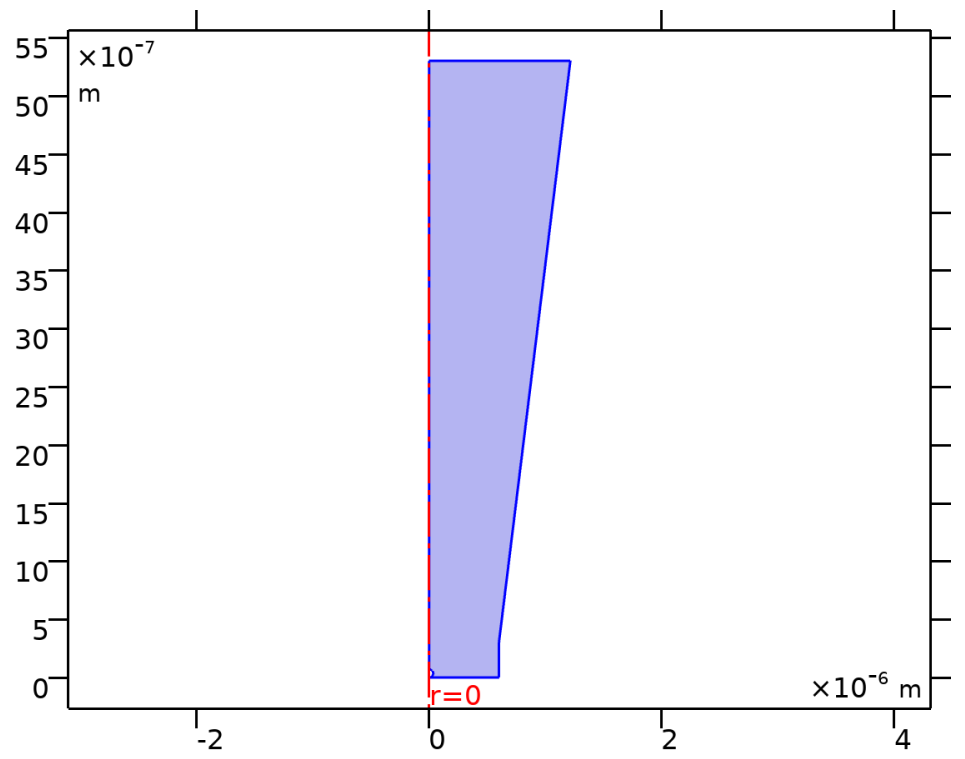

Charge Conservation 1

SELECTION

Geometric entity level

Selection

Domain

Geometry geom1: Dimension 2: All domains

EQUATIONS

$$
\begin{aligned}
& \mathbf{E}=-\nabla V \\
& \nabla \cdot\left(\epsilon_{0} \epsilon_{r} \mathbf{E}\right)=\rho_{v} \\
& \cdots \ldots . . . \cdots
\end{aligned}
$$

Constitutive relation D-E

SETTINGS

Description

Value 


\begin{tabular}{|l|l|}
\hline Description & Value \\
\hline Dielectric model & Relative permittivity \\
\hline Relative permittivity & User defined \\
\hline Relative permittivity & $\{\{78.5,0,0\},\{0,78.5,0\},\{0,0,78.5\}\}$ \\
\hline
\end{tabular}

Coordinate system selection

SETTINGS

\begin{tabular}{l|l} 
Description & Value \\
\hline Coordinate system & Global coordinate system
\end{tabular}

Shape functions

\begin{tabular}{|l|l|l|l|l|l|}
\hline Name & Shape function & Unit & Description & Shape frame & Selection \\
\hline V & Lagrange (Quadratic) & $\mathrm{V}$ & Electric potential & Spatial & Domain 1 \\
\hline $\mathrm{V}$ & Lagrange (Quadratic) & $\mathrm{V}$ & Electric potential & Material & Domain 1 \\
\hline $\mathrm{V}$ & Lagrange (Quadratic) & $\mathrm{V}$ & Electric potential & Geometry & Domain 1 \\
\hline $\mathrm{V}$ & Lagrange (Quadratic) & $\mathrm{V}$ & Electric potential & Mesh & Domain 1 \\
\hline
\end{tabular}

Weak expressions

\begin{tabular}{|l|l|l|l|}
\hline Weak expression & Integration order & Integration frame & Selection \\
\hline- & & & \\
\hline $\begin{array}{l}2^{*}\left(\text { es.Dr*test }(\mathrm{Vr})+\mathrm{es} . \mathrm{Dz}{ }^{*} \operatorname{test}(\mathrm{V} z)\right)^{*} \text { es.d*pi } \\
{ }_{r}\end{array}$ & 4 & Spatial & Domain 1 \\
\hline
\end{tabular}

\subsubsection{Axial Symmetry 1}

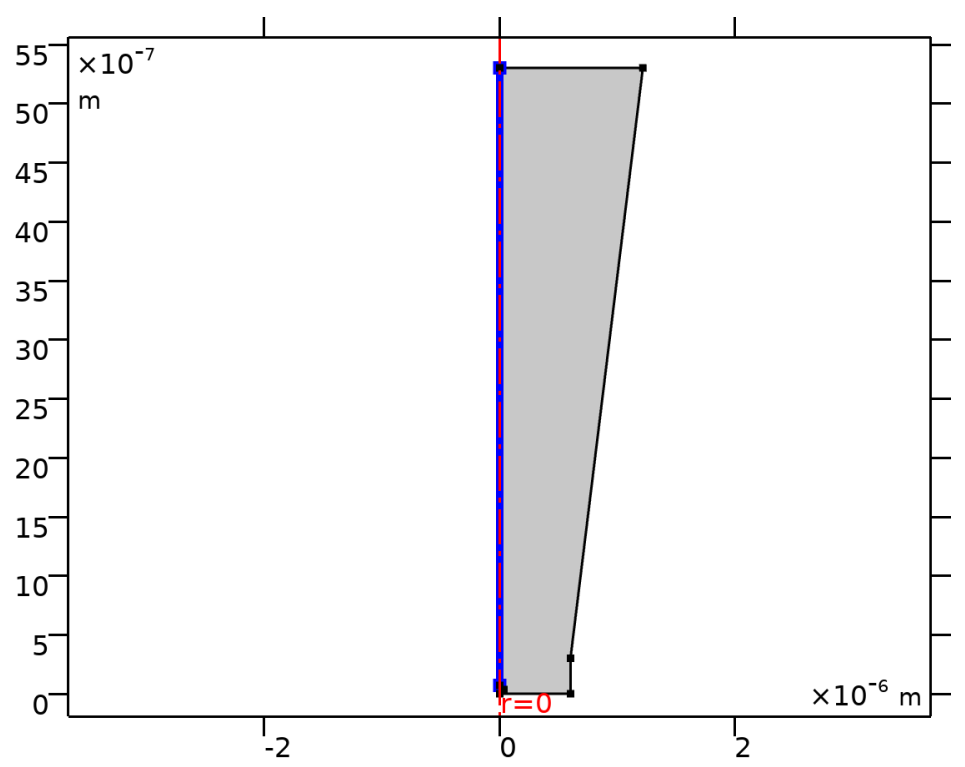

Axial Symmetry 1

SELECTION 


\begin{tabular}{|l|l|}
\hline Geometric entity level & Boundary \\
\hline Selection & Geometry geom1: Dimension 1: All boundaries
\end{tabular}

\subsubsection{Zero Charge 1}

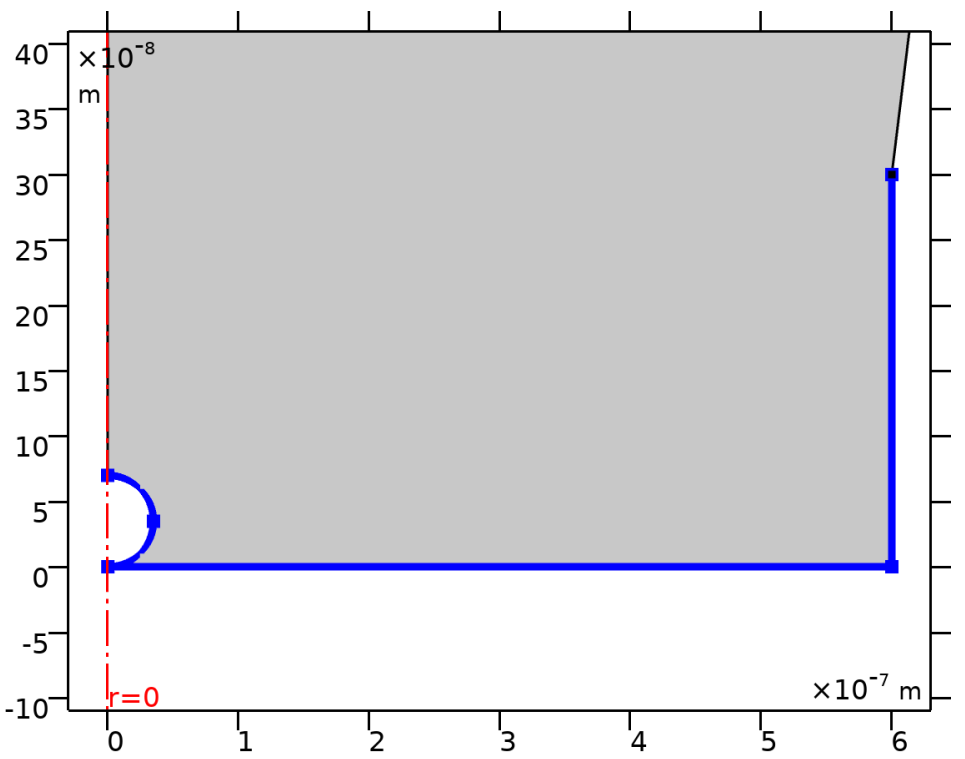

Zero Charge 1

\section{SELECTION}

Geometric entity level Boundary

Selection Geometry geom1: Dimension 1: All boundaries

EQUATIONS

$$
\mathbf{n} \cdot \mathbf{D}=0
$$

Variables

\begin{tabular}{|l|l|l|l|l|l|}
\hline Name & Expression & Unit & Description & Selection & Details \\
\hline es.nD & 0 & $\mathrm{C} / \mathrm{m}^{2}$ & Surface charge density & Boundaries 3-4, 6-7 & + operation \\
\hline
\end{tabular}

Shape functions

\begin{tabular}{|c|c|c|c|c|c|c|}
\hline Name & Shape function & Unit & Description & Shape frame & Selection & Details \\
\hline V & $\begin{array}{l}\text { Lagrange } \\
\text { (Quadratic) }\end{array}$ & V & Electric potential & Spatial & No boundaries & Slit \\
\hline V & $\begin{array}{l}\text { Lagrange } \\
\text { (Quadratic) }\end{array}$ & V & Electric potential & Material & No boundaries & Slit \\
\hline V & $\begin{array}{l}\text { Lagrange } \\
\text { (Quadratic) }\end{array}$ & V & Electric potential & Geometry & No boundaries & Slit \\
\hline V & $\begin{array}{l}\text { Lagrange } \\
\text { (Quadratic) }\end{array}$ & $\mathrm{V}$ & Electric potential & Mesh & No boundaries & Slit \\
\hline
\end{tabular}




\subsubsection{Initial Values 1}

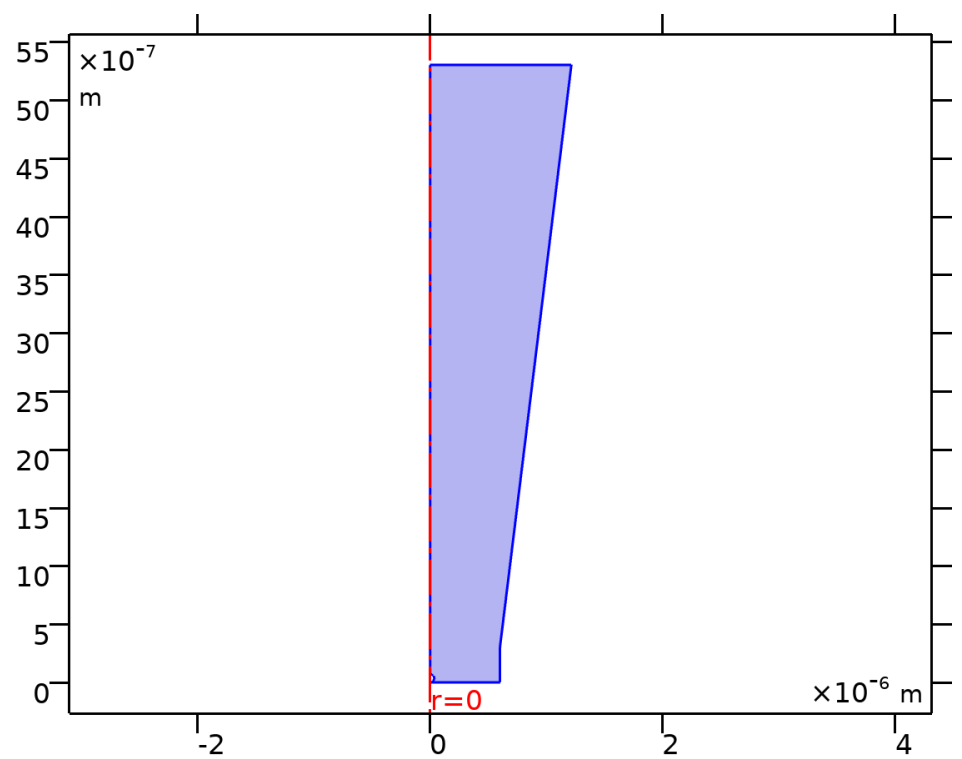

Initial Values 1

\section{SELECTION}

Geometric entity level Domain

Selection

SETTINGS

\section{Description Value}

Electric potential

\subsubsection{Ground 1}

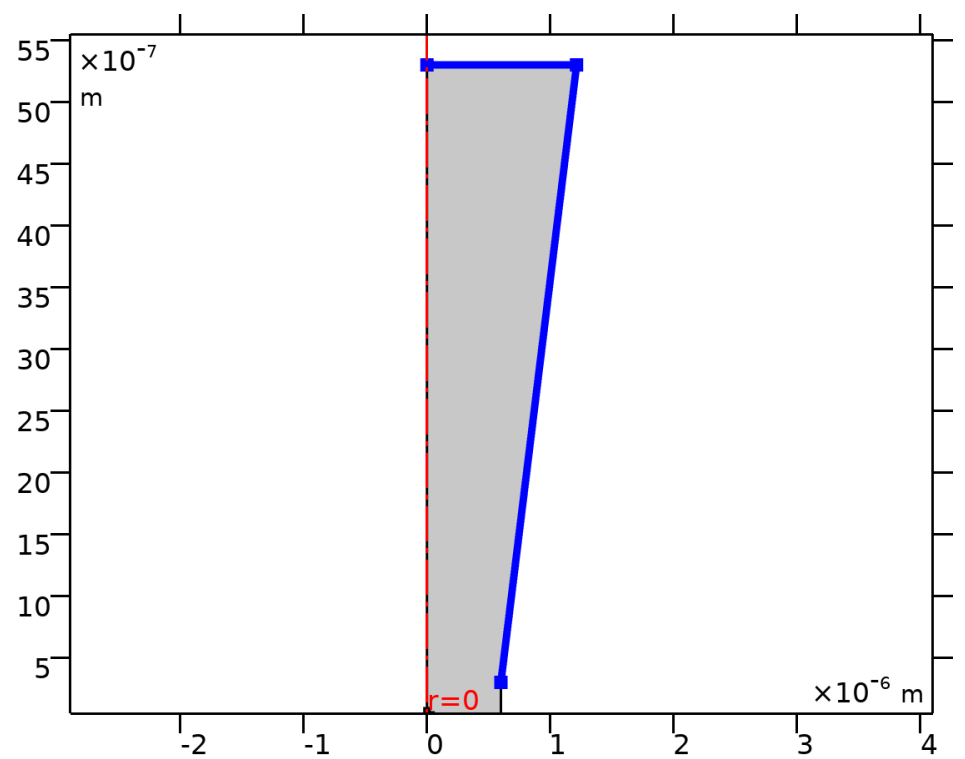

Ground 1 
SELECTION

Geometric entity level Boundary

Selection

Geometry geom1: Dimension 1: Boundaries 2, 5

EQUATIONS

$V=0$

Variables

\begin{tabular}{|l|l|l|l|l|l|}
\hline Name & Expression & Unit & Description & Selection & Details \\
\hline es.nD & $\begin{array}{l}\text { es.unr*down(es.Dr)+es.unphi*do } \\
\text { wn(es.Dphi)+es.unz*down(es.Dz) }\end{array}$ & $\mathrm{C} / \mathrm{m}^{2}$ & $\begin{array}{l}\text { Surface charge } \\
\text { density }\end{array}$ & Boundaries 2, 5 & + operation \\
\hline
\end{tabular}

Constraints

\begin{tabular}{|l|l|l|l|l|}
\hline Constraint & Constraint force & Shape function & Selection & Details \\
\hline$-\mathrm{V}$ & test(-V) & Lagrange (Quadratic) & Boundaries 2, 5 & Elemental \\
\hline
\end{tabular}

\subsubsection{Space Charge Density 1}

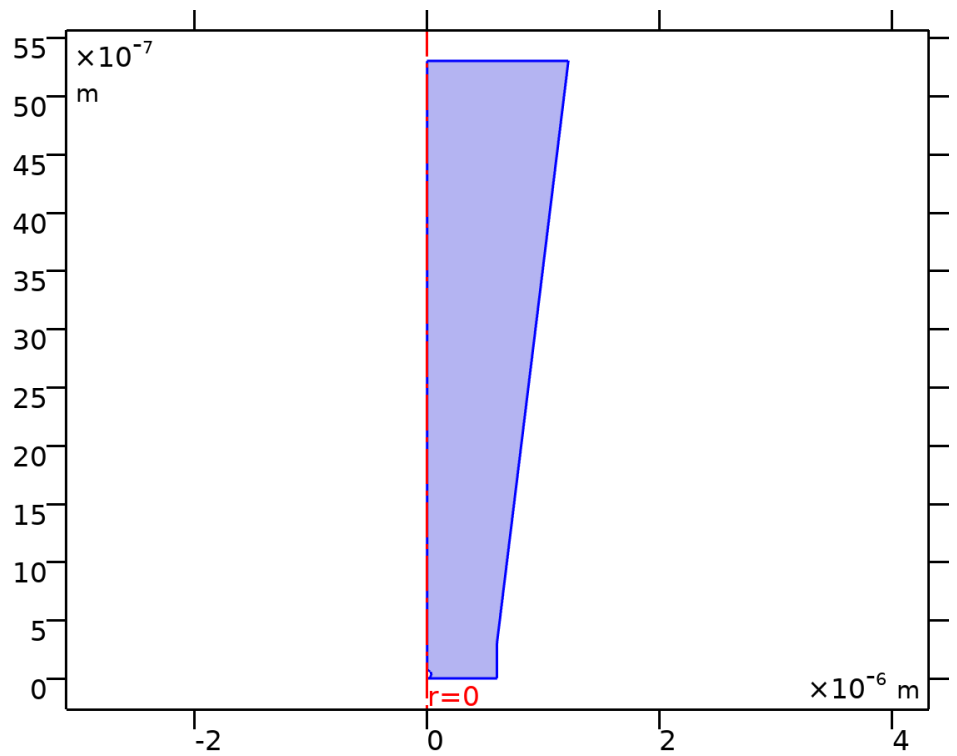

Space Charge Density 1

SELECTION

Geometric entity level Domain

Selection

Geometry geom1: Dimension 2: Domain 1

EQUATIONS

$\nabla \cdot \mathbf{D}=\rho_{\mathrm{v}}$

Coordinate system selection

SETTINGS 


\begin{tabular}{|l|l|}
\hline Description & Value \\
\hline Coordinate system & Global coordinate system \\
\hline
\end{tabular}

Variables

\begin{tabular}{|l|l|l|l|l|l|}
\hline Name & Expression & Unit & Description & Selection & Details \\
\hline es.scd1.rhoq & F_const*(H-ClO4) & $\mathrm{C} / \mathrm{m}^{3}$ & $\begin{array}{l}\text { Space charge } \\
\text { density }\end{array}$ & Domain 1 \\
\hline es.rhoq & es.scd1.rhoq & $\mathrm{C} / \mathrm{m}^{3}$ & $\begin{array}{l}\text { Space charge } \\
\text { density }\end{array}$ & Domain 1 & + operation \\
\hline
\end{tabular}

Weak expressions

Weak expression

$-2 *$ es.scd1.rhoq*test(V)*es.d*pi*r

\section{Integration order}

4

\section{Integration frame}

Spatial
Selection

Domain 1

\subsection{TRANSPORT OF DILUTED SPECIES}

USED PRODUCTS

COMSOL Multiphysics

Batteries \& Fuel Cells Module

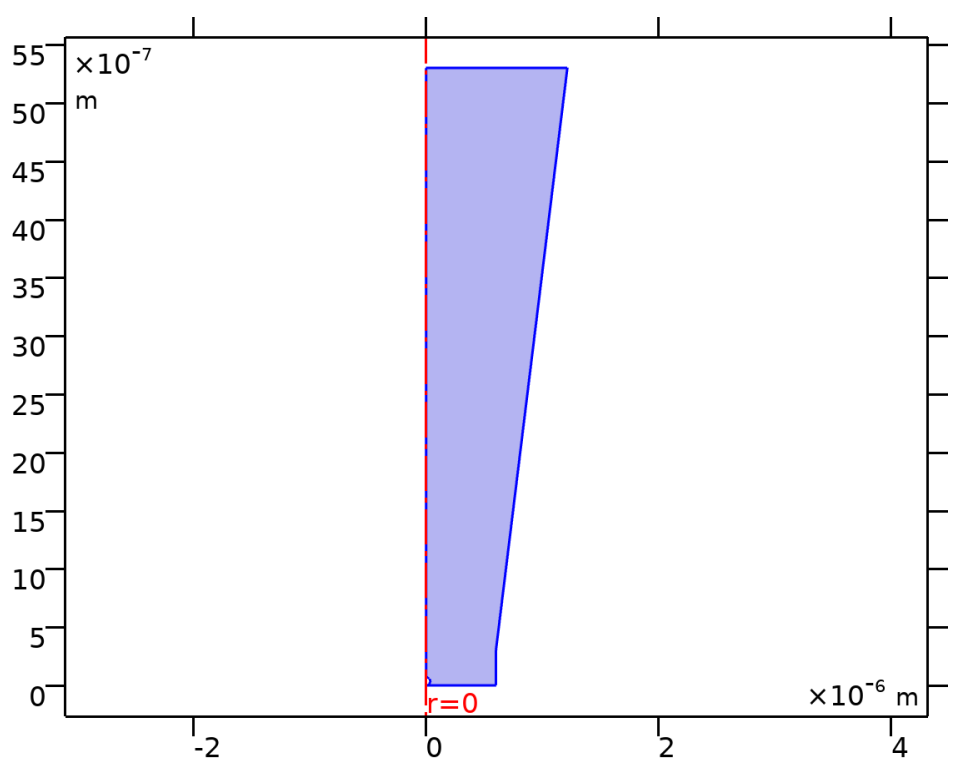

Transport of Diluted Species

\section{SELECTION}

Geometric entity level Domain

Selection

Geometry geom1: Dimension 2: All domains

EQUATIONS

$$
\begin{aligned}
& \nabla \cdot \mathbf{J}_{i}=R_{i} \\
& \mathbf{J}_{i}=-D_{i} \nabla c_{i}-z_{i} u_{m, j} F c_{i} \nabla V
\end{aligned}
$$




\subsubsection{Interface settings}

Discretization

SETTINGS

\begin{tabular}{|l|l|}
\hline Description & Value \\
\hline Concentration & Linear
\end{tabular}

Transport mechanisms

SETTINGS

\begin{tabular}{|l|l|}
\hline Description & Value \\
\hline Convection & Off \\
\hline Migration in electric field & On \\
\hline Mass transfer in porous media & Off \\
\hline
\end{tabular}

\subsubsection{Transport Properties 1}

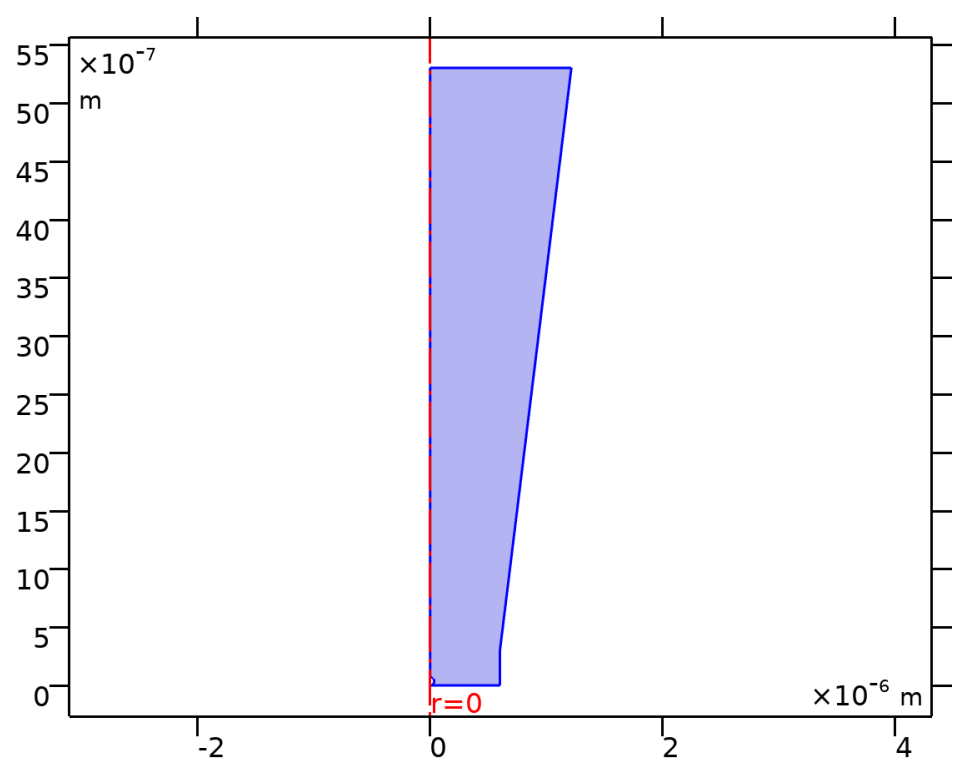

Transport Properties 1

SELECTION

Geometric entity level Domain

Selection Geometry geom1: Dimension 2: All domains

EQUATIONS

$$
\begin{aligned}
& \nabla \cdot \mathbf{J}_{i}=R_{i} \\
& \dddot{\mathbf{J}_{i}}=-D_{i} \nabla c_{i}-z_{i} u_{\mathrm{m}, i} F c_{i} \nabla V
\end{aligned}
$$

Diffusion 


\begin{tabular}{|l|l|}
\hline Description & Value \\
\hline Material & None \\
\hline Diffusion coefficient & User defined \\
\hline Diffusion coefficient & $\{\{\mathrm{DH}, 0,0\},\{0, \mathrm{DH}, 0\},\{0,0, \mathrm{DH}\}\}$ \\
\hline Diffusion coefficient & User defined \\
\hline Diffusion coefficient & $\{\{\mathrm{DH} 2,0,0\},\{0, \mathrm{DH} 2,0\},\{0,0, \mathrm{DH} 2\}\}$ \\
\hline Diffusion coefficient & User defined \\
\hline Diffusion coefficient & $\{\{\mathrm{DClO} 4,0,0\},\{0, \mathrm{DClO} 4,0\},\{0,0, \mathrm{DClO} 4\}\}$ \\
\hline
\end{tabular}

Migration in electric field

SETTINGS

\begin{tabular}{|l|l|}
\hline Description & Value \\
\hline Electric potential & Electric potential (es) \\
\hline Mobility & Nernst - Einstein relation \\
\hline Charge number & $\{1,0,-1\}$ \\
\hline
\end{tabular}

Coordinate system selection

SETTINGS

\begin{tabular}{|l|l|}
\hline Description & Value \\
\hline Coordinate system & Global coordinate system
\end{tabular}

Model input

\begin{tabular}{l} 
SETTINGS \\
\begin{tabular}{|l|l|}
\hline Description & Value \\
\hline Temperature & User defined \\
\hline Temperature & $293.15[\mathrm{~K}]$ \\
\hline
\end{tabular} \\
\hline
\end{tabular}

Shape functions

\begin{tabular}{|l|l|l|l|l|l|}
\hline Name & Shape function & Unit & Description & Shape frame & Selection \\
\hline $\mathrm{H}$ & Lagrange (Linear) & $\mathrm{mol} / \mathrm{m}^{3}$ & Concentration & Spatial & Domain 1 \\
\hline $\mathrm{H} 2$ & Lagrange (Linear) & $\mathrm{mol} / \mathrm{m}^{3}$ & Concentration & Spatial & Domain 1 \\
\hline $\mathrm{ClO} 4$ & Lagrange (Linear) & $\mathrm{mol} / \mathrm{m}^{3}$ & Concentration & Spatial & Domain 1 \\
\hline
\end{tabular}

Weak expressions

\begin{tabular}{|c|c|c|c|}
\hline Weak expression & Integration order & Integration frame & Selection \\
\hline $\begin{array}{l}2^{*}\left(\mathrm{tds} . d f l u x \_H r^{*} \text { test}(\mathrm{Hr})+\mathrm{tds} . d f l u x \_H z * t e\right. \\
\operatorname{st}(\mathrm{Hz}))^{*} \mathrm{pi}^{*} r\end{array}$ & 2 & Spatial & Domain 1 \\
\hline 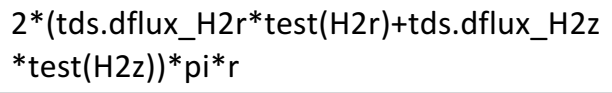 & 2 & Spatial & Domain 1 \\
\hline $\begin{array}{l}2 * \text { (tds.dflux_ClO4r*test }(\mathrm{ClO} 4 \mathrm{r})+\text { tds.dflux_ } \\
\text { ClO4z*test }(\mathrm{ClO} 4 \mathrm{z}))^{*} \mathrm{pi}^{*}{ }_{\mathrm{r}}\end{array}$ & 2 & Spatial & Domain 1 \\
\hline
\end{tabular}




\begin{tabular}{|c|c|c|c|}
\hline Weak expression & Integration order & Integration frame & Selection \\
\hline $\begin{array}{l}2^{*} \text { tds.z_H*F_const*H*((- } \\
\text { tds.um_Hrr*d(tds.V,r)- } \\
\text { tds.um_Hrz*d(tds.V,z))*test }(H r)+(- \\
\text { tds.um_Hzr*d(tds.V,r)- } \\
\text { tds.um_Hzz*d(tds.V,z))*test(Hz))*pi*r }\end{array}$ & 2 & Spatial & Domain 1 \\
\hline $\begin{array}{l}2 * \text { tds.z_H2*F_const*H2*((- } \\
\text { tds.um_H2rr*d(tds.V,r)- } \\
\text { tds.um_H2rz*d(tds.V,z) })^{*} \text { test }(H 2 r)+(- \\
\text { tds.um_H2zr*d(tds.V,r)- } \\
\text { tds.um_H2zz*d(tds.V,z))*test }(H 2 z))^{*} \text { pi*r }^{*}\end{array}$ & 2 & Spatial & Domain 1 \\
\hline $\begin{array}{l}2 * \text { tds.z_ClO4*F_const*ClO4*((- } \\
\text { tds.um_ClO4rr*d(tds.V,r)- } \\
\text { tds.um_ClO4rz*d(tds.V,z))*test(ClO4r)+(- } \\
\text { tds.um_ClO4zr*d(tds.V,r)- } \\
\text { tds.um_ClO4zz*d(tds.V,z) }) * \operatorname{test}(\mathrm{ClO} 4 \mathrm{z}))^{*} \mathrm{p} \\
\text { i*r }^{*}\end{array}$ & 2 & Spatial & Domain 1 \\
\hline $\begin{array}{l}2 * \text { tds.streamline*(isScalingSystemDomai } \\
\mathrm{n}==0)^{*} \mathrm{pi}^{*} \mathrm{r}\end{array}$ & 2 & Spatial & Domain 1 \\
\hline $\begin{array}{l}2 * \text { tds.crosswind*(isScalingSystemDomain } \\
==0) * \mathrm{pi}^{*} \mathrm{r}\end{array}$ & 4 & Spatial & Domain 1 \\
\hline
\end{tabular}

\subsubsection{Axial Symmetry 1}

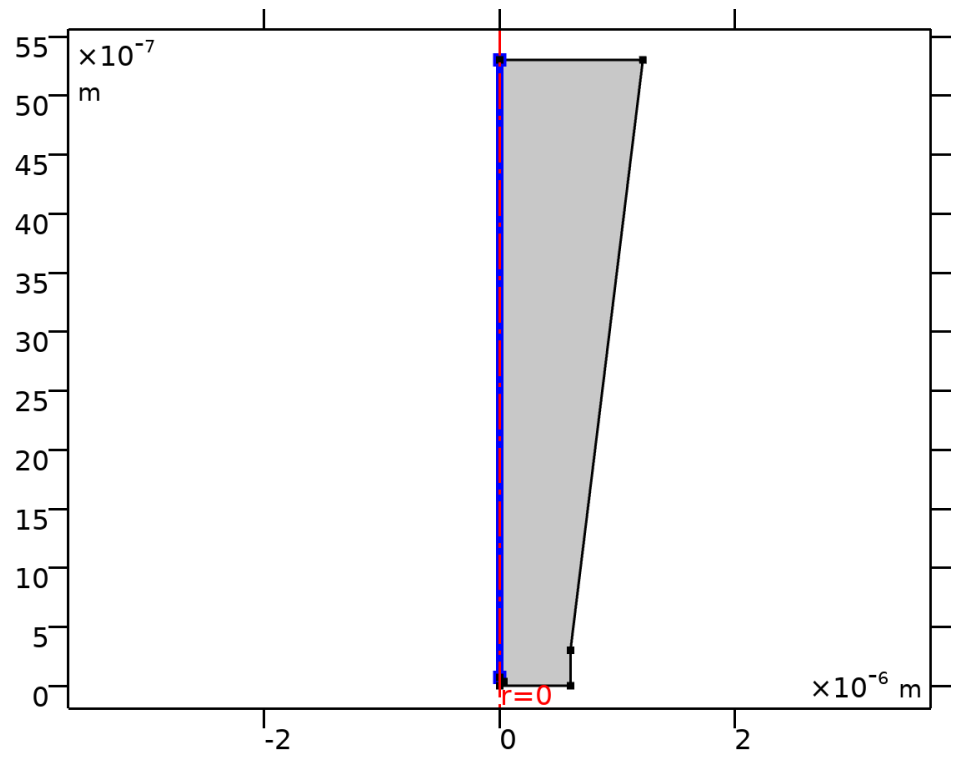

Axial Symmetry 1

\section{SELECTION}

Geometric entity level Boundary

Selection

Geometry geom1: Dimension 1: All boundaries 


\subsubsection{No Flux 1}

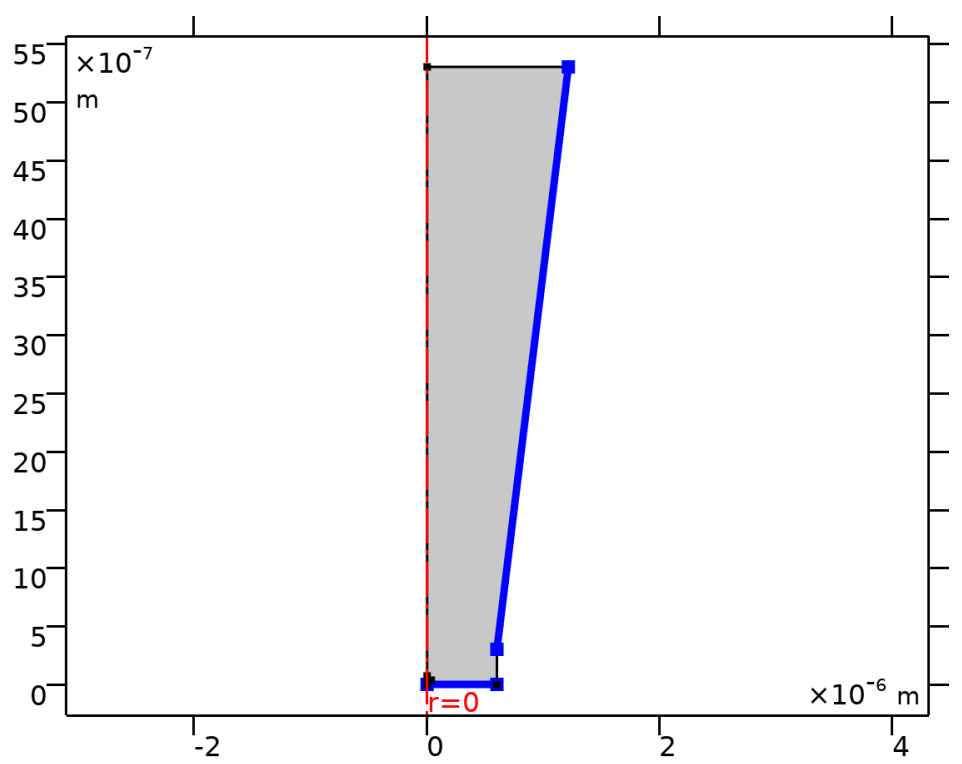

No Flux 1

\section{SELECTION}

Geometric entity level Boundary

Selection Geometry geom1: Dimension 1: All boundaries

EQUATIONS

$$
-\mathbf{n} \cdot\left(\mathbf{J}_{i}+\mathbf{u} c_{i}\right)=0
$$

\subsubsection{Initial Values 1}

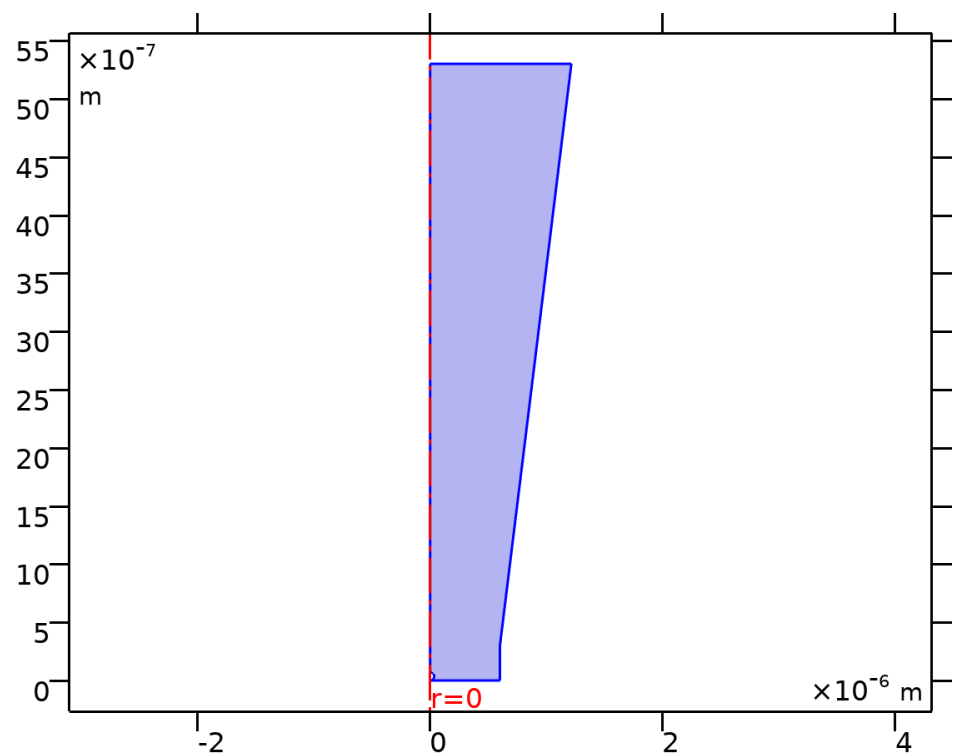

Initial Values 1 
SELECTION

Geometric entity level Domain

Selection

Geometry geom1: Dimension 2: All domains

Initial values

SETTINGS

\section{Description Value \\ Concentration $\{$ cbulk, 0, cbulk\}}

\subsubsection{Flux 1}

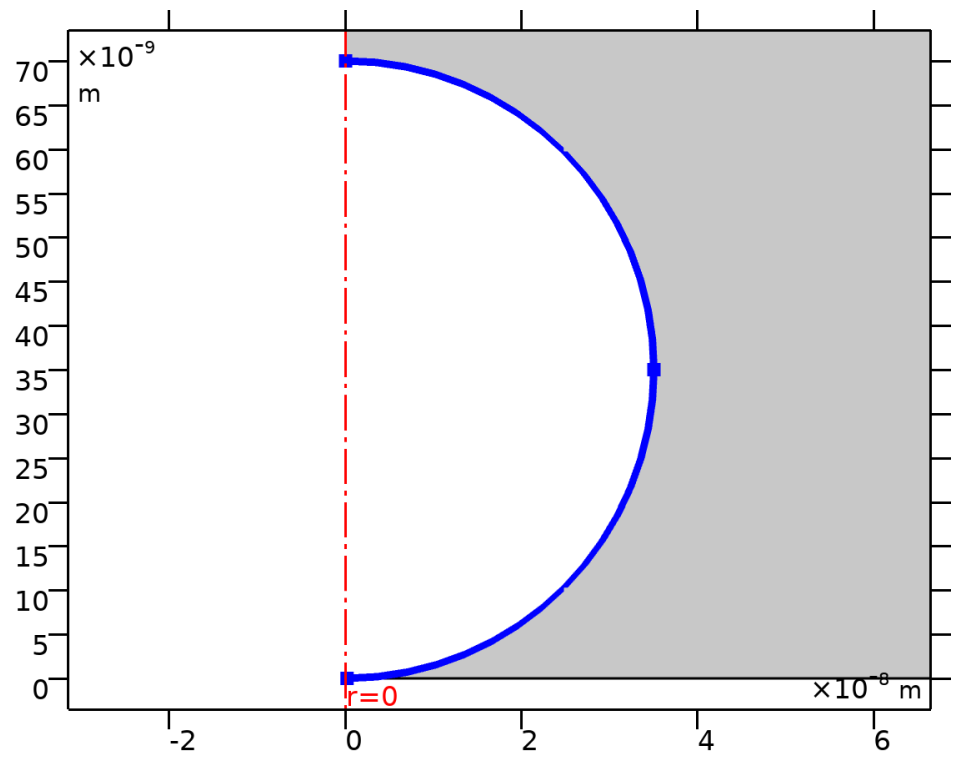

Flux 1

\section{SELECTION}

Geometric entity level Boundary

Selection

Geometry geom1: Dimension 1: Boundaries 6-7

EQUATIONS

$$
-\mathbf{n} \cdot\left(\mathbf{J}_{i}+\mathbf{u} c_{i}\right)=N_{0, j}
$$

Inward flux

\section{SETTINGS}

\begin{tabular}{l|l} 
Description & Value \\
\hline Flux type & General inward flux \\
\hline Species H & Off \\
\hline Species H2 & On \\
\hline Species ClO4 & Off
\end{tabular}


Weak expressions

\begin{tabular}{|l|l|l|l|}
\hline Weak expression & Integration order & Integration frame & Selection \\
\hline 0 & 2 & Spatial & Boundaries 6-7 \\
\hline tds.ntflux_H*test(H2)*pi*r & 2 & Spatial & Boundaries 6-7 \\
\hline 0 & 2 & Spatial & Boundaries 6-7 \\
\hline
\end{tabular}

\subsubsection{Concentration 1}

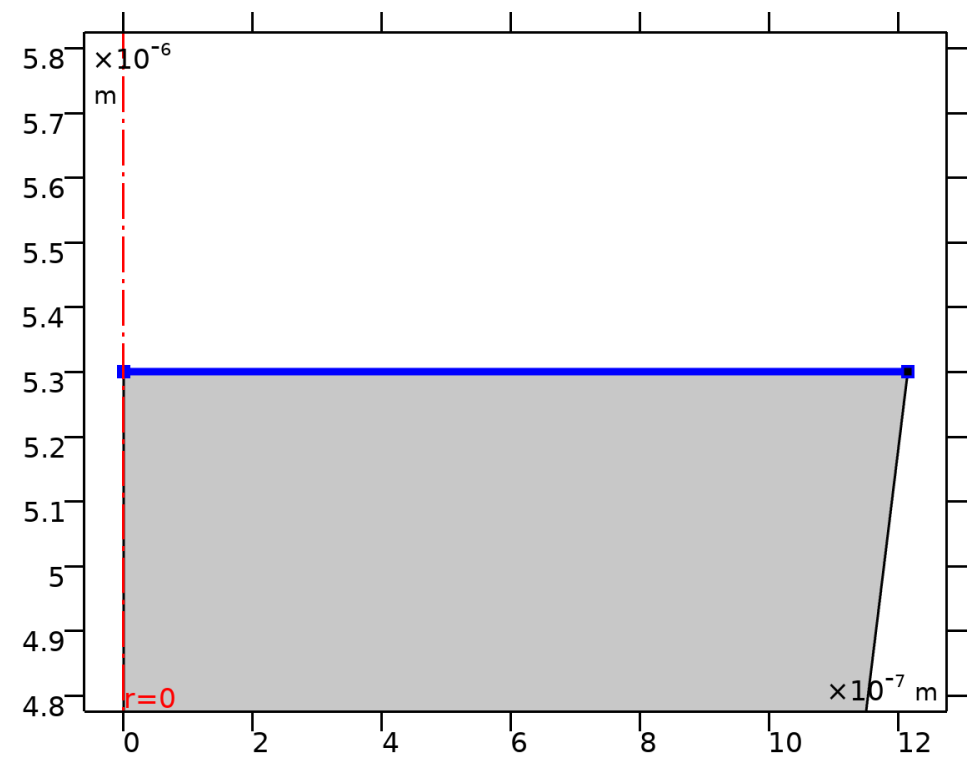

Concentration 1

SELECTION

Geometric entity level Boundary

Selection $\quad$ Geometry geom1: Dimension 1: Boundary 2

EQUATIONS

$c_{i}=c_{0, j}$

Concentration

SETTINGS

\begin{tabular}{l|l} 
Description & Value \\
\hline Species H & On \\
\hline Species H2 & On \\
\hline Species ClO4 & On \\
\hline Concentration & $\{$ cbulk, 0, cbulk\} \\
\hline
\end{tabular}

Variables

\begin{tabular}{|l|l|l|l|l|}
\hline Name & Expression & Unit & Description & Selection \\
\hline tds.c0_H & cbulk & $\mathrm{mol} / \mathrm{m}^{3}$ & Concentration & Boundary 2 \\
\hline & & & $\mathrm{S} 17$ \\
\hline
\end{tabular}




\begin{tabular}{|l|l|l|l|l|}
\hline Name & Expression & Unit & Description & Selection \\
\hline tds.cO_H2 & 0 & $\mathrm{~mol} / \mathrm{m}^{3}$ & Concentration & Boundary 2 \\
\hline tds.cO_ClO4 & cbulk & $\mathrm{mol} / \mathrm{m}^{3}$ & Concentration & Boundary 2 \\
\hline
\end{tabular}

Constraints

\begin{tabular}{|c|c|c|c|c|}
\hline Constraint & Constraint force & Shape function & Selection & Details \\
\hline$-\mathrm{H}+\mathrm{tds} . \mathrm{cO} \_\mathrm{H}$ & test(-H+tds.cO_H) & Lagrange (Linear) & Boundary 2 & Elemental \\
\hline$-\mathrm{H} 2+\mathrm{tds} . \mathrm{cO} \_\mathrm{H} 2$ & test(-H2+tds.c0_H2) & Lagrange (Linear) & Boundary 2 & Elemental \\
\hline -ClO4+tds.c0_ClO4 & test(-ClO4+tds.c0_ClO4) & Lagrange (Linear) & Boundary 2 & Elemental \\
\hline
\end{tabular}

\subsubsection{Concentration 2}

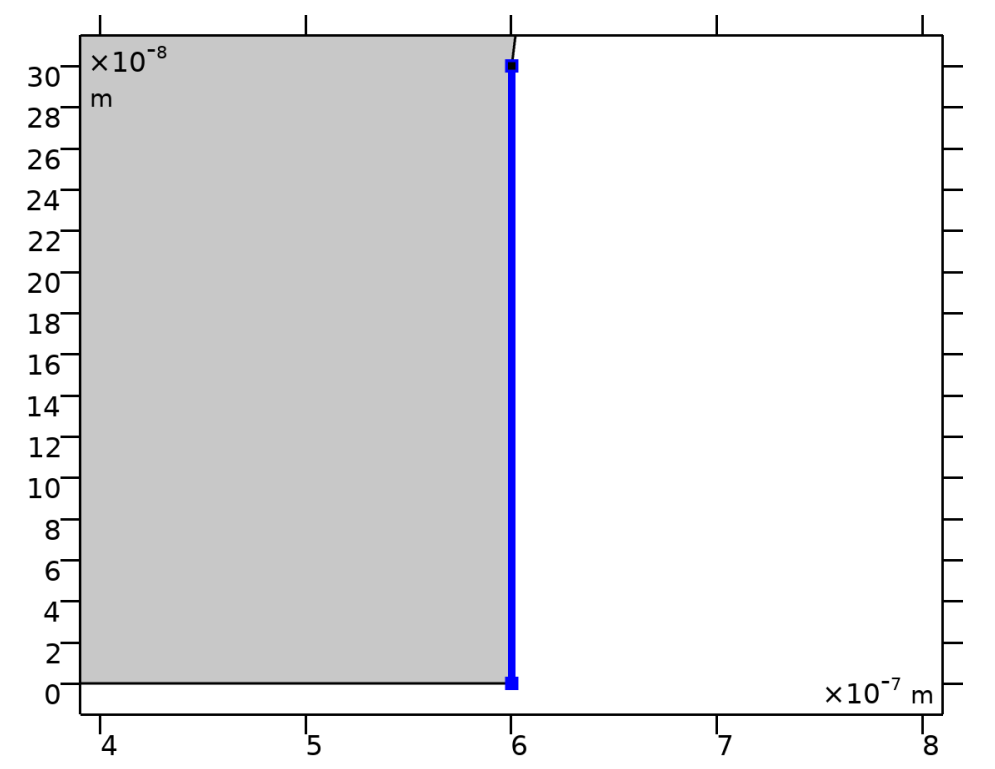

Concentration 2

\section{SELECTION}

Geometric entity level Boundary

Selection Geometry geom1: Dimension 1: Boundary 4

EQUATIONS

$c_{i}=c_{0, j}$

SETTINGS

\begin{tabular}{|l|l|}
\hline Description & Value \\
\hline Concentration & $\{$ cbulk, 0, cbulk $\}$ \\
\hline
\end{tabular}

Concentration

SETTINGS

\begin{tabular}{l|l} 
Description & Value \\
\hline Species H & Off
\end{tabular}




\begin{tabular}{|l|l|}
\hline Description & Value \\
\hline Species $\mathrm{H} 2$ & On \\
\hline Species $\mathrm{ClO} 4$ & Off \\
\hline
\end{tabular}

Variables

\begin{tabular}{|l|l|l|l|l|}
\hline Name & Expression & Unit & Description & Selection \\
\hline tds.CO_H2 & 0 & $\mathrm{~mol} / \mathrm{m}^{3}$ & Concentration & Boundary 4 \\
\hline
\end{tabular}

Constraints

\begin{tabular}{|l|l|l|l|l|}
\hline Constraint & Constraint force & Shape function & Selection & Details \\
\hline 0 & 0 & Lagrange (Linear) & Boundary 4 & Elemental \\
\hline$-\mathrm{H} 2+\mathrm{tds} . \mathrm{CO}+\mathrm{H} 2$ & test(-H2+tds.c0_H2) & Lagrange (Linear) & Boundary 4 & Elemental \\
\hline 0 & 0 & Lagrange (Linear) & Boundary 4 & Elemental \\
\hline
\end{tabular}

\subsubsection{Concentration 3}

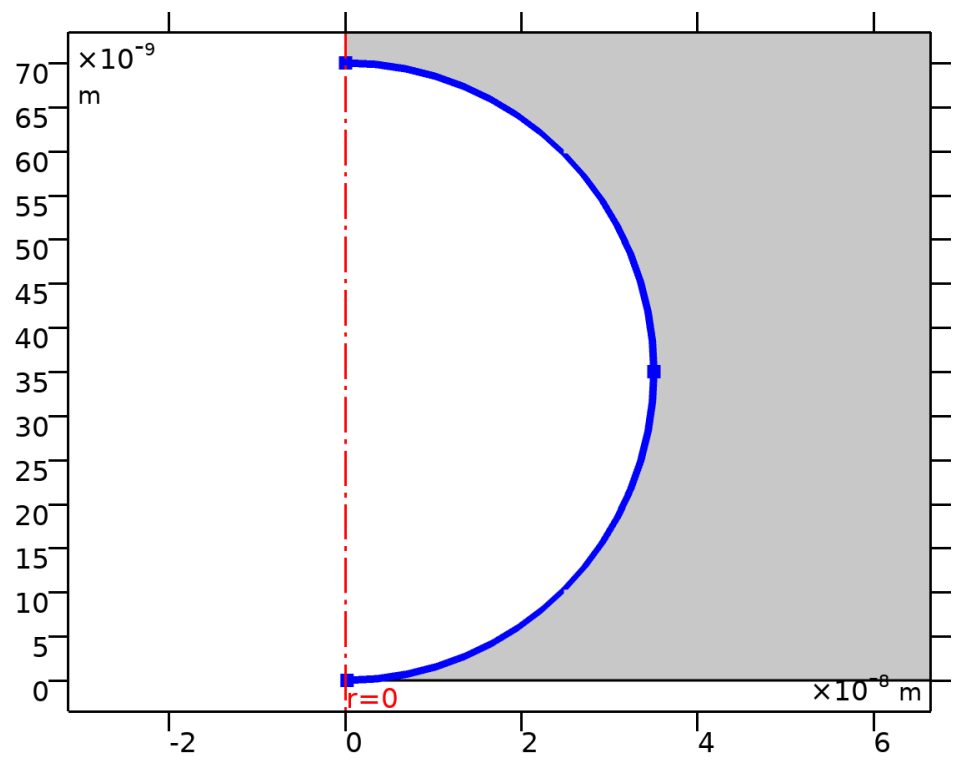

Concentration 3

SELECTION

Geometric entity level Boundary

Selection Geometry geom1: Dimension 1: Boundaries 6-7

EQUATIONS

$c_{i}=c_{0, j}$

Concentration

SETTINGS

Description Value 


\begin{tabular}{|l|l|}
\hline Description & Value \\
\hline Species $\mathrm{H}$ & On \\
\hline Species $\mathrm{H} 2$ & Off \\
\hline Species $\mathrm{ClO} 4$ & Off \\
\hline Concentration & $\{0,0,0\}$ \\
\hline
\end{tabular}

Variables

\begin{tabular}{|l|l|l|l|l|}
\hline Name & Expression & Unit & Description & Selection \\
\hline tds.c0_H & 0 & $\mathrm{~mol} / \mathrm{m}^{3}$ & Concentration & Boundaries 6-7 \\
\hline
\end{tabular}

Constraints

\begin{tabular}{|l|l|l|l|l|}
\hline Constraint & Constraint force & Shape function & Selection & Details \\
\hline$-\mathrm{H}+\mathrm{tds} . \mathrm{CO} \_\mathrm{H}$ & test(-H+tds.c0_H) & Lagrange (Linear) & Boundaries 6-7 & Elemental \\
\hline 0 & 0 & Lagrange (Linear) & Boundaries 6-7 & Elemental \\
\hline 0 & 0 & Lagrange (Linear) & Boundaries 6-7 & Elemental \\
\hline
\end{tabular}




\section{Limiting Current}

COMPUTATION INFORMATION

\begin{tabular}{|l|l|}
\hline Computation time & $12 \mathrm{~s}$ \\
\hline CPU & Intel64 Family 6 Model 158 Stepping 13, 6 cores \\
\hline Operating system & Windows 10 \\
\hline
\end{tabular}

\subsection{STATIONARY}

STUDY SETTINGS

\begin{tabular}{|l|l|}
\hline Description & Value \\
\hline Include geometric nonlinearity & Off \\
\hline
\end{tabular}

PHYSICS AND VARIABLES SELECTION

\begin{tabular}{|l|l|}
\hline Physics interface & Discretization \\
\hline Electrostatics (es) & physics \\
\hline Transport of Diluted Species (tds) & physics \\
\hline
\end{tabular}

MESH SELECTION

\begin{tabular}{|l|l|}
\hline Geometry & Mesh \\
\hline Geometry 1 (geom1) & mesh1 \\
\hline
\end{tabular}

\subsection{SOLVER CONFIGURATIONS}

\subsubsection{Solution 147}

Compile Equations: Stationary (st1)

STUDY AND STEP

\begin{tabular}{l|l} 
Description & Value \\
\hline Use study & $\underline{\text { Limiting Current }}$ \\
\hline Use study step & $\underline{\text { Stationary }}$ \\
\hline
\end{tabular}

Dependent Variables 1 (v1)

GENERAL

\begin{tabular}{l|l}
\hline Description & Value \\
\hline Defined by study step & Stationary \\
\hline
\end{tabular}

Concentration (comp1.ClO4) (comp1_ClO4)

GENERAL

\begin{tabular}{|l|l|}
\hline Description & Value \\
\hline Field components & comp1.ClO4 \\
\hline Internal variables & $\{$ comp1.uflux.ClO4, comp1.dflux.ClO4\} \\
\hline
\end{tabular}


Concentration (comp1.H) (comp1_H)

GENERAL

\begin{tabular}{l|l}
\hline Description & Value \\
\hline Field components & comp1.H \\
\hline Internal variables & \{comp1.uflux.H, comp1.dflux.H
\end{tabular}

Concentration (comp1.H2) (comp1_H2)

GENERAL

\begin{tabular}{l|l} 
Description & Value \\
\hline Field components & comp1.H2 \\
\hline Internal variables & \{comp1.uflux.H2, comp1.dflux.H2\}
\end{tabular}

Electric potential (comp1.V) (comp1_V)

GENERAL

\begin{tabular}{|l|l|}
\hline Description & Value \\
\hline Field components & comp1.V \\
\hline
\end{tabular}

Stationary Solver 1 (s1)

GENERAL

\begin{tabular}{|l|l|} 
Description & Value \\
\hline Defined by study step & Stationary \\
\hline
\end{tabular}

Fully Coupled 1 (fc1)

GENERAL

\begin{tabular}{l|l|} 
Description & Value \\
\hline Linear solver & Direct 1 \\
\hline METHOD AND TERMINATION
\end{tabular}

\begin{tabular}{|l|l|}
\hline Description & Value \\
\hline Initial damping factor & 0.01 \\
\hline Minimum damping factor & $1.0 \mathrm{E}-6$ \\
\hline Maximum number of iterations & 50 \\
\hline
\end{tabular}

